\title{
Restrictive Cardiomyopathy Secondary to Hydroxychloroquine Therapy
}

VINAYAK A. MANOHAR, MD, Resident; KEVIN G. MODER, MD, Staff Consultant, Division of Rheumatology, Department of Internal Medicine; WILLIAM D. EDWARDS, MD, Staff Consultant, Department of Laboratory Medicine and Pathology; KYLE W. KLARICH, MD, Staff Consultant, Division of Cardiovascular Diseases, Department of Internal Medicine, Mayo Clinic, Rochester, Minnesota, USA. Address reprint requests to Dr. K.W. Klarich, 200 First Street SW, Rochester, MN 55902. J Rheumatol 2009;36:472-3; doi:10.3899/jrheum.080305

A 64-year-old woman with a history of systemic lupus erythematosus, treated for more than 10 years with prednisone and hydroxychloroquine, presented with severe progressive dyspnea on exertion. She had no other significant medical history. Examination revealed an S3 gallop. Transthoracic echocardiogram (TTE) demonstrated a $46 \%$ ejection fraction $(\mathrm{EF})$ with generalized left ventricular hypokinesis, grade 3/4 diastolic dysfunction, and elevated left ventricular filling pressure. Left ventricular chamber size was normal, with concentric wall thickening. The myocardium had a hyper-refractile appearance (Figure 1). Serum protein electrophoresis and abdominal fat aspiration with Congo red staining were negative. Endomyocardial biopsy revealed sarcoplasmic clearing and vacuolization on light microscopy and myelinoid and curvilinear bodies on electron microscopy (Figure 2). Hydroxychloroquine toxicity was diagnosed and the medication was discontinued. The patient was asymptomatic within 9 months. Followup TTE revealed a $74 \% \mathrm{EF}$ with normal left ventricular systolic function and grade 2/4 diastolic dysfunction. Wall thickness had normalized.

Hydroxychloroquine cardiomyopathy is characterized by biatrial enlargement, biventricular thickening, and systolic and diastolic dysfunction ${ }^{1,2}$. Hydroxychloroquine induces the formation of sarcoplasmic myelinoid and curvilinear bodies that lead to myocyte dysfunction. Sarcoplasmic accumulation of myelinoid and curvilinear bodies expands the myocytes and interferes with their function. As a result, ventricular wall thickness is increased ${ }^{3,4}$. Definitive diagnosis requires electron microscopy of endomyocardial biopsy tis-

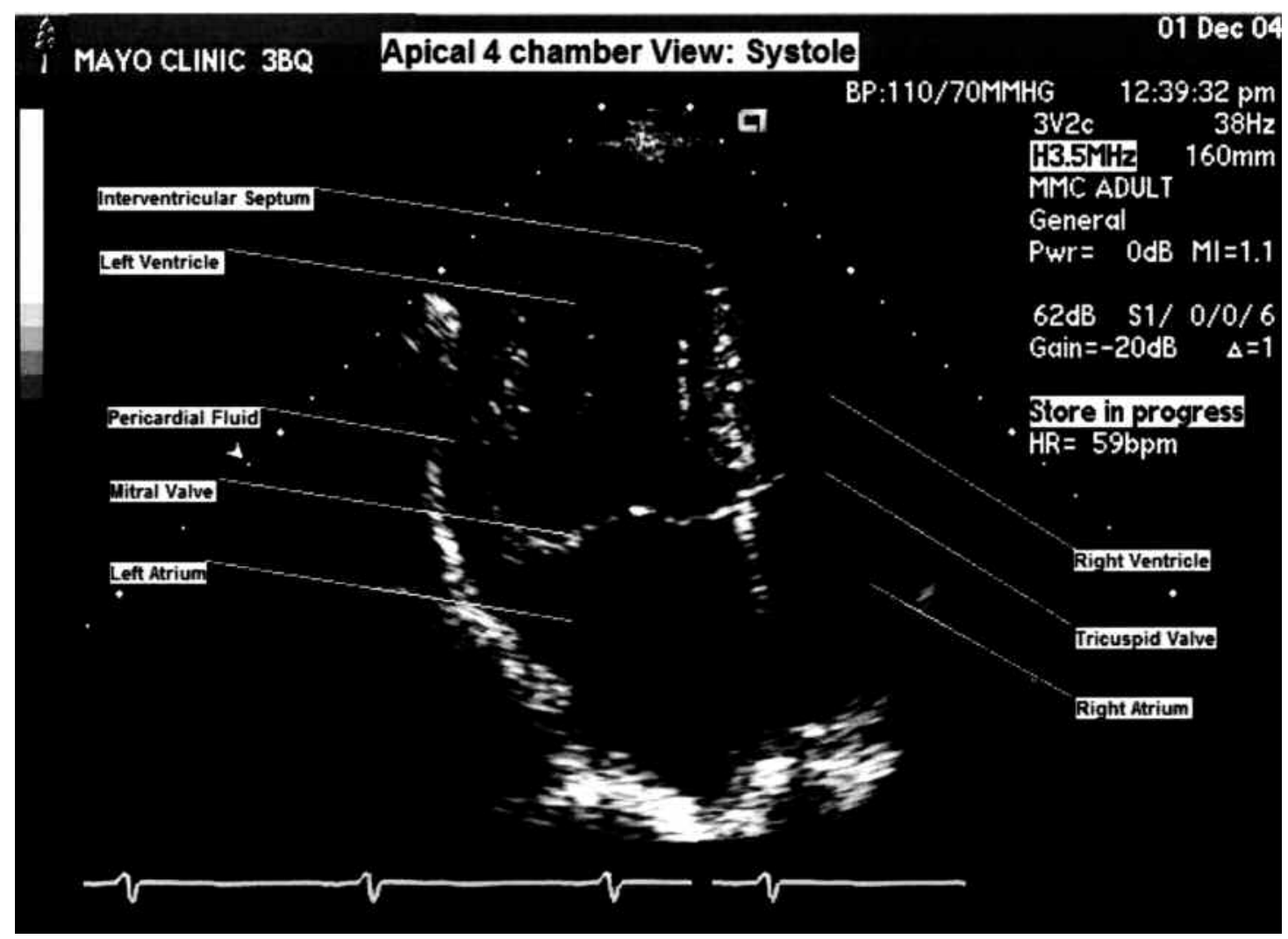

Figure 1. Hyper-refractile appearance of the myocardium. 


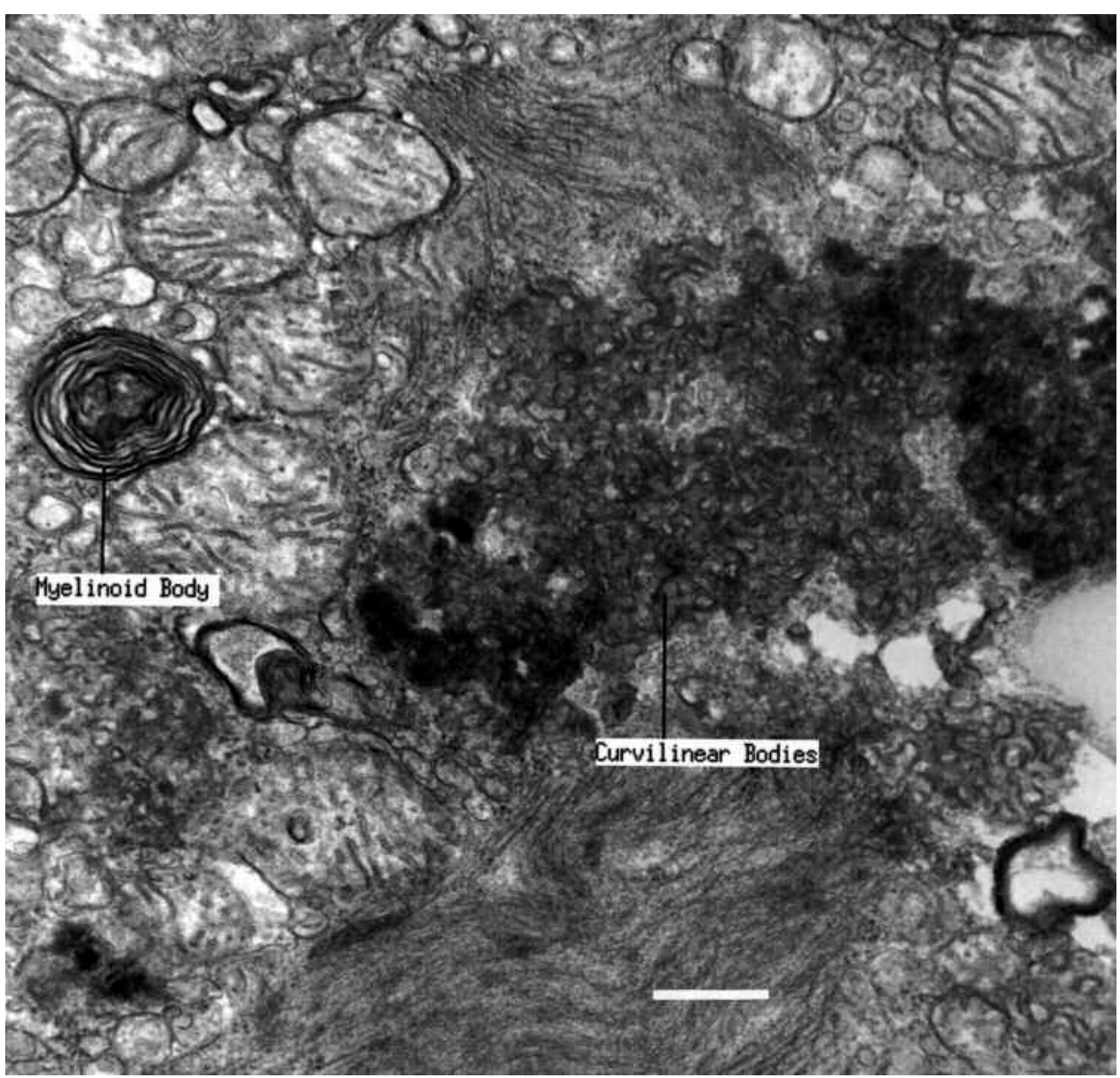

Figure 2. Endomyocardial biopsy revealed sarcoplasmic clearing and vacuolization on light microscopy and myelinoid and curvilinear bodies on electron microscopy. $\mathrm{Bar}=500 \mathrm{~nm}$.

sue revealing unique curvilinear bodies in the sarcoplasm ${ }^{1}$. These represent abnormal lysosomes replete with phospholipids and glucose. Withdrawal of hydroxychloroquine leads to symptomatic and echocardiographic improvement.

\section{REFERENCES}

1. Keating RJ, Bhatia S, Amin S, Williams A, Sinak LJ, Edwards WD. Hydroxychloroquine-induced cardiotoxicity in a 39-year-old woman with systemic lupus erythematosus and systolic dysfunction. J Am Soc Echocardiogr 2005;18:981e1-e5.

2. Roos JM, Aubry MC, Edwards WD. Chloroquine cardiotoxicity:
Clinicopathologic features in three patients and comparison with three patients with Fabry disease. Cardiovasc Pathol 2002;11:277-283

3. Iglesias Cubero G, Rodriguez Reguero JJ, Rojo Ortega JM. Restrictive cardiomyopathy caused by chloroquine. Br Heart J 1993;69:451-2.

4. Cervera A, Espinosa G, Font J, Ingelmo M. Cardiac toxicity secondary to long term treatment with chloroquine. Ann Rheum Dis 2001;60:301. 